\title{
SYMMETRIC SPECTRAL FACTORISATION OF SELF-ADJOINT RATIONAL MATRIX FUNCTIONS
}

\author{
G.J. Groenewald and M.A. Petersen
}

\begin{abstract}
For a self-adjoint rational matrix function, not necessarily analytic at infinity, the existence of a right (symmetric) spectral factorisation is described in terms of a given left spectral factorisation. The formula for the right spectral factor is given in terms of the formula for the given left spectral factor. All formulas are based on a special realisation of a rational matrix function, which is different from ones that have been used before.
\end{abstract}

\section{INTRODUCTION}

It is well known that Wiener-Hopf and spectral factorisations of matrix and operator-valued functions have numerous applications in analysis and electrical engineering. Applications include singular integral equations (see $[6,10,13]$ ), Toeplitz operators (see [8]), algebraic Riccati equations (see [14] and [15]) and model reduction for linear systems (see $[\mathbf{1}, \mathbf{2}]$ ). Moreover, in the analysis of $H_{\infty}$-control problems, spectral factorisation plays an important role (see for example, $[\mathbf{1 1}, \mathbf{7}]$ ). In the latter theories, the matrix functions are in general assumed to be rational, that is, their entries are quotients of polynomials.

In this paper, we use the state space method (see [5]), which depends on the notion of realisation and allows one to reduce problems concerning rational matrix functions to ones in linear algebra involving constant matrices. By a realisation for a rational matrix function $W$ (which is analytic and invertible at $\infty$ ) we mean a representation for $W$ of the form

$$
W(\lambda)=I+C(\lambda-A)^{-1} B
$$

where $A$ is an $n \times n$ matrix and $B$ and $C$ are $n \times m$ and $m \times n$ matrices, respectively. Here $I$ denotes the $m \times m$ identity matrix.

In [3], Ball and Ran showed how the state space method may be applied to rational matrix functions (of the form (1)) which are analytic and invertible at infinity. We follow a similar program as in [3] (see also [12]), but with a different representation. Indeed,

Received 3rd September, 1996

The second author was partially supported by a grant from the FRD in South Africa.

Copyright Clearance Centre, Inc. Serial-fee code: 0004-9729/97 \$A2.00+0.00. 
in our case, the analysis is based on the following representation of the given rational matrix function:

$$
W(\lambda)=I+C(\lambda G-A)^{-1} B
$$

Here the matrix $G$ is of the same order as $A$, the matrices $B$ and $C$ are as before and the pencil $\lambda G-A$ is regular on the unit circle $|\lambda|=1$.

In this article, we extend a factorisation result of $[3]$ to the general case. Given a signed antispectral factorisation

$$
W(\lambda)=Y_{-}^{*}(\lambda)\left(\begin{array}{cc}
I_{p} & 0 \\
0 & -I_{q}
\end{array}\right) Y_{-}(\lambda)
$$

with respect to the unit circle, where $Y_{-}(\lambda)$ and its inverse are given in realisation form, the aim is to find necessary and sufficient conditions for the existence of a signed spectral factorisation

$$
W(\lambda)=X_{+}^{*}(\lambda)\left(\begin{array}{cc}
I_{p} & 0 \\
0 & -I_{q}
\end{array}\right) X_{+}(\lambda),
$$

and give an explicit formula for a spectral factor $X_{+}(\lambda)$ and its inverse in realisation form.

The paper consists of three sections, including the introduction. The second section summarises the preliminary notions and results. Section 3 contains the main factorisation result.

\section{Preliminaries}

Firstly, we give terminology and notation. By a Cauchy contour $\gamma$, we mean the positively oriented boundary of a bounded Cauchy domain in $\mathbb{C}$. Such a contour consists of a finite number of non-intersecting closed rectifiable Jordan curves. The set of points inside $\gamma$ is called the inner domain of $\gamma$ and will be denoted by $\Delta_{+}$. The outer domain of $\gamma$ is the set $\Delta_{-}=\mathbb{C}_{\infty} \backslash \bar{\Delta}_{+}$. By convention $0 \in \Delta_{+}$and by definition $\infty \in \Delta_{-}$.

Next, we consider operator pencils. Let $X$ be a complex Banach space and let $G$ and $A$ be bounded linear operators on $X$. For $\lambda \in \mathbb{C}$, the expression $\lambda G-A$ is called a (linear) operator pencil on $X$. Given a non-empty subset $\Delta$ of the Riemann sphere $\mathbb{C}_{\infty}$, we say that $\lambda G-A$ is $\Delta-$ regular if $\lambda G-A$ (or just $G$ if $\lambda=\infty$ ) is invertible for each $\lambda \in \Delta$. The spectrum of $\lambda G-A$, denoted by $\sigma(G, A)$, is the subset of $\mathbb{C}_{\infty}$ determined by the following properties: $\infty \in \sigma(G, A)$ if and only if $G$ is not invertible, and $\sigma(G, A) \cap \mathbb{C}$ consists of all those $\lambda \in \mathbb{C}$ for which $\lambda G-A$ is not invertible. Its complement (in $\mathbb{C}_{\infty}$ ) is the resolvent set of $\lambda G-A$, denoted by $\rho(G, A)$. 
Let $W(\lambda)$ be a rational $m \times m$ matrix function with neither a pole nor a zero on $\gamma$. Then $W(\lambda)$ admits a (right) Wiener-Hopf factorisation relative to $\gamma$, that is, $W(\lambda)$ factorises as

$$
W(\lambda)=W_{-}(\lambda) D(\lambda) W_{+}(\lambda), \quad \lambda \in \gamma,
$$

where $W_{+}$and $W_{-}$are rational $m \times m$ matrix functions, $W_{+}$has neither a pole nor a zero on $\Delta_{+} \cup \gamma$ and $W_{-}$has neither a pole nor a zero on $\Delta_{-} \cup \gamma$ (which includes the point $\infty$ ), and

$$
D(\lambda)=\operatorname{diag}\left(\lambda^{\kappa_{j}}\right)_{j=1}^{m} .
$$

Here $\kappa_{1} \leqslant \kappa_{2} \leqslant \ldots \leqslant \kappa_{m}$ are integers, which are uniquely determined by $W$ (and $\gamma$ ), and are called the (right) factorisation indices of $W$ relative to $\gamma$ (see, for example, [6]). The factorisation is called a (right) canonical Wiener-Hopf factorisation if and only if the indices $\kappa_{1}, \ldots, \kappa_{m}$ are all zero. If $W$ admits such a factorisation, then $\operatorname{det} W(\lambda) \neq 0$ for each $\lambda \in \gamma$. In general, this condition is only necessary but not sufficient for the existence of a canonical factorisation. We refer to a (left) Wiener-Hopf factorisation if in (3) the order of the factors are interchanged.

Throughout this paper, we shall consider the representation of the rational matrix function $W$ of the form (see [9]):

$$
W(\lambda)=I+C(\lambda G-A)^{-1} B, \quad|\lambda|=1 .
$$

The square matrices $G$ and $A$ are both of order $n$ say, and $B$ and $C$ are matrices of sizes $n \times m$ and $m \times n$, respectively. Also, we assume that the pencil $\lambda G-A$ is regular on the unit circle $\mathbb{T}=\{\lambda:|\lambda|=1\}$.

Put $A^{\times}=A-B C$. Then $\operatorname{det} W(\lambda) \neq 0$ if and only if $\lambda G-A^{\times}$is $\mathbb{T}$-regular. In this case,

$$
W(\lambda)^{-1}=I-C\left(\lambda G-A^{\times}\right)^{-1} B, \quad|\lambda|=1,
$$

(see $[\mathbf{9}])$.

Next, we state [13, Theorem 2.3]. We shall present an application of this factorisation theorem in the next section. The result involves left and right canonical factorisation of rational matrix functions. A thorough discussion of these concepts can be found in $[\mathbf{4}]$ and [3]. For instance, it is shown how to compute realisations for the factors $W_{-}$and $W_{+}$of a right canonical factorisation $W=W_{-} W_{+}$, if one is given a realisation $W(\lambda)=I+C(\lambda-A)^{-1} B$ for $\mathrm{W}$. Furthermore, it is known how to compute a right canonical Wiener-Hopf factorisation $W(\lambda)=W_{-}(\lambda) W_{+}(\lambda)$ in terms of a given left canonical factorisation $W(\lambda)=Y_{+}(\lambda) Y_{-}(\lambda)$ where the left factors are given by $Y_{+}(\lambda)=$ $I+C_{+}\left(\lambda-A_{+}\right)^{-1} B_{+}$and $Y_{-}(\lambda)=I+C_{-}\left(\lambda-A_{-}\right)^{-1} B_{-}$. However, as in $[\mathbf{1 3}$, Section 
2], we assume that the factors $Y_{+}$and $Y_{-}$of a left canonical Wiener-Hopf factorisation are given by the more general realisations $Y_{+}(\lambda)=I+C_{+}\left(\lambda G_{+}-A_{+}\right)^{-1} B_{+}$and $Y_{-}(\lambda)=I+C_{-}\left(\lambda G_{-}-A_{-}\right)^{-1} B_{-}$. We then state a necessary and sufficient condition for the existence of a right canonical Wiener-Hopf factorisation $W(\lambda)=W_{-}(\lambda) W_{+}(\lambda)$. In this case, we provide explicit formulas for the factors $W_{-}$and $W_{+}$in terms of the realisations of $Y_{+}$and $Y_{-}$. Let the inner and outer domain of the unit circle $\mathbb{T}$ be denoted by $\mathbb{D}_{+}$and $\mathbb{D}_{-}$, respectively. The result is as follows.

Theorem 2.1. Suppose that the rational $m \times m$ matrix function $W(\lambda)$ (not necessarily analytic and invertible at $\infty$ ) has a left canonical Wiener-Hopf factorisation with respect to $\mathbb{T}$, that is, $W(\lambda)$ factorises as

$$
W(\lambda)=Y_{+}(\lambda) Y_{-}(\lambda)
$$

where

$$
Y_{+}(\lambda)=I_{m}+C_{+}\left(\lambda G_{+}-A_{+}\right)^{-1} B_{+}
$$

and

$$
Y_{-}(\lambda)=I_{m}+C_{-}\left(\lambda G_{-}-A_{-}\right)^{-1} B_{-}
$$

Set $A_{-}^{\times}:=A_{-}-B_{-} C_{-}$and $A_{+}^{\times}:=A_{+}-B_{+} C_{+}$. Assume that $\lambda G_{-}-A_{-}$and $\lambda G_{-}-A_{-}^{\times}$are $n_{-} \times n_{-}$matrix pencils which are $\left(\mathbb{D}_{-} \cup \mathbb{T}\right)$-regular and that $\lambda G_{+}-A_{+}$ and $\lambda G_{+}-A_{+}^{\times}$are $n_{+} \times n_{+}$matrix pencils which are $\left(\mathbb{D}_{+} \cup \mathbb{T}\right)$-regular. Let $U$ and $T$ denote the unique solutions of the Lyapunov equations

$$
A_{-}^{\times} U G_{+}^{-} G_{-} U A_{+}^{\times}=B_{-} C_{+}
$$

and

$$
G_{+} T A_{-}-A_{+} T G_{-}=B_{+} C_{-}
$$

Then $W$ has a right canonical Wiener-Hopf factorisation if and only if the $n_{-} \times n_{-}$ matrix $I_{n_{-}}-G_{-} U G_{+} T$ is invertible, or equivalently, if and only if the $n_{-} \times n_{-}$matrix $I_{n_{-}}-U G_{+} T G_{-}$is invertible, or equivalently, if and only if the $n_{+} \times n_{+}$matrix $I_{n_{+}}$ $G_{+} T G_{-} U$ is invertible, or equivalently, if and only if the $n_{+} \times n_{+}$matrix $I_{n_{+}}-$ $T G_{-} U G_{+}$is invertible. In this case, the factors $W_{-}(\lambda)$ and $W_{+}(\lambda)$ for a right canonical Wiener-Hopf factorisation are given by the formulas

(8) $W_{-}(\lambda)=I+\left[C_{+} T G_{-}+C_{-}\right]\left(\lambda G_{--}-A_{-}\right)^{-1}\left[I_{n_{-}}-G_{-} U G_{+} T\right]^{-1}\left[-G_{-} U B_{+}+B_{-}\right]$, 
and

(9) $W_{+}(\lambda)=I+\left[C_{+}+C_{-} U G_{+}\right]\left[I_{n_{+}}-T G_{-} U G_{+}\right]^{-1}\left(\lambda G_{+}-A_{+}\right)^{-1}\left[B_{+}-G_{+} T B_{-}\right]$.

Their inverses are given by

$W_{-}(\lambda)^{-1}=I-\left[C_{+} T G_{-}+C_{-}\right]\left[I_{n_{-}}-U G_{+} T G_{-}\right]^{-1}\left(\lambda G_{-}-A_{-}^{\times}\right)^{-1}\left[-G_{-} U B_{+}+B_{-}\right]$,

and

(11) $W_{+}(\lambda)^{-1}=I-\left[C_{+}+C_{-} U G_{+}\right]\left(\lambda G_{+}-A_{+}^{\times}\right)^{-1}\left[I_{n_{+}}-G_{+} T G_{-} U\right]^{-1}\left[B_{+}-G_{+} T B_{-}\right]$.

3. Antispectral versus spectral factorisation With Respect to the UNIT CIRCLE

We assume that $W(\lambda)$ is a rational $m \times m$ matrix function which is analytic and invertible on the unit circle $\{\lambda:|\lambda|=1\}$ such that $W(1 / \bar{\lambda})^{*}=W(\lambda)$. In general, in this section we shall use $W^{*}$ to denote the function $W^{*}(\lambda)=W(1 / \bar{\lambda})^{*}$. Observe, that for a rational matrix function $W$, we have that $W=W^{*}$ if and only if $W(\lambda)$ is self-adjoint for $|\lambda|=1$. Since by hypothesis, $W(\lambda)$ is self-adjoint and invertible on the unit circle, it follows that $W\left(e^{i \tau}\right)$ has non-zero real eigenvalues, that is, $W\left(e^{i \tau}\right)$ must have a constant number (say $p$ ) of positive eigenvalues and $q=m-p$ of negative eigenvalues for all real $\tau$.

By a signed antispectral factorisation of $W$ (with respect to the unit circle) we mean a factorisation of the form:

$$
W(\lambda)=Y_{-}^{*}(\lambda)\left(\begin{array}{cc}
I_{p} & 0 \\
0 & -I_{q}
\end{array}\right) Y_{-}(\lambda),
$$

where $Y_{-}(\lambda)$ is analytic and invertible on the exterior of the unit disc $\overline{\mathbb{D}}_{-}=\{\lambda:|\lambda| \geqslant$ $1\}$. By a signed spectral factorisation of $W$ (with respect to the unit circle) we mean a factorisation of the form:

$$
W(\lambda)=X_{+}^{*}(\lambda)\left(\begin{array}{cc}
I_{p} & 0 \\
0 & -I_{q}
\end{array}\right) X_{+}(\lambda)
$$

where $X_{+}(\lambda)$ is analytic and invertible on the closed unit disc $\overline{\mathbb{D}}_{+}=\{\lambda:|\lambda| \leqslant 1\}$.

The problem which we consider here, may be viewed as a symmetrised version of the one analysed in [13]. Indeed, given a signed antispectral factorisation

$$
W(\lambda)=Y_{-}^{*}(\lambda)\left(\begin{array}{cc}
I_{p} & 0 \\
0 & -I_{q}
\end{array}\right) Y_{-}(\lambda),
$$


find necessary and sufficient conditions for the existence of a signed spectral factorisation. In this case, give an explicit formula for a spectral factor $X_{+}(\lambda)$, and its inverse. In order to achieve this, we need the fact that a function $W$, which is selfadjoint and invertible on the unit circle, has a signed spectral factorisation if and only if it has a right canonical Wiener-Hopf factorisation with respect to the unit circle. In particular, if $W(\lambda)=W_{-}(\lambda) W_{+}(\lambda)$ is a right canonical factorisation then $W(\lambda)=W^{*}(\lambda)=W_{+}^{*}(\lambda) W_{-}^{*}(\lambda)$ is also one. However, it is known that such factorisations are unique up to a constant invertible factor; hence $W_{-}(\lambda)=W_{+}^{*}(\lambda) K$, where $K$ is some non-singular $m \times m$ matrix, and $W(\lambda)=W_{+}^{*}(\lambda) K W_{+}(\lambda)$. Upon substituting $\lambda=1$, we get that $K=W_{+}^{*}(1)^{-1} W(1) W_{+}(1)^{-1}$ is self-adjoint (and invertible), so as before $K$ has $p$ positive and $q$ negative eigenvalues. Then $K$ factors as:

$$
K=E^{*}\left(\begin{array}{cc}
I_{p} & 0 \\
0 & -I_{q}
\end{array}\right) E
$$

for some invertible $m \times m$ matrix $E$. Thus

$$
W(\lambda)=X_{+}^{*}(\lambda)\left(\begin{array}{cc}
I_{p} & 0 \\
0 & -I_{q}
\end{array}\right) X_{+}(\lambda)
$$

is a signed spectral factorisation, where $X_{+}(\lambda)=E W_{+}(\lambda)$. By using these observations and Theorem 2.1, we obtain an analogous result for signed spectral factorisation.

THEOREM 3.1. Suppose that the rational $m \times m$ matrix function $W(\lambda)=$ $W^{*}(\lambda)$ has a signed antispectral factorisation

$$
W(\lambda)=Y_{-}^{*}(\lambda)\left(\begin{array}{cc}
I_{p} & 0 \\
0 & -I_{q}
\end{array}\right) Y_{-}(\lambda)
$$

where

$$
Y_{-}(\lambda)=Y_{-}(\infty)\left[I_{m}+C_{-}\left(\lambda G_{-}-A_{-}\right)^{-1} B_{-}\right] .
$$

We may asssume that $\lambda G_{-}-A_{-}$and $\lambda G_{-}-A_{-}^{\times}$are $n_{-} \times n_{-}$matrices with spectra in the open unit disc $\mathbb{D}_{+}$. Here $A_{-}^{\times}:=A_{-}-B_{-} C_{-}$. We also assume that $Y_{-}(\infty)$ and $Y_{-}^{*}(\infty)=Y_{-}(0)^{*}$ are invertible, so $W(\infty)$ and $W(0)=W(\infty)^{*}$ are invertible. We denote by $\psi$ the Hermitian matrix

$$
\psi=Y_{-}(\infty)^{*}\left(\begin{array}{cc}
I_{p} & 0 \\
0 & -I_{q}
\end{array}\right) Y_{-}(\infty) .
$$

Let $U$ and $T$ denote the unique solutions of the Stein equations

$$
A_{-}^{\times} U G_{-}^{*-1}\left(A_{-}^{\times}\right)^{*}-G_{-} U=-B_{-} \psi^{-1} B_{-}^{*},
$$


and

$$
A_{-}^{*} G_{-}^{*-1} T A_{-}-T G_{-}=C_{-}^{*} \psi C_{-} .
$$

Then $W$ has a signed spectral factorisation if and only if the $n_{+} \times n_{+}$matrix $I_{n_{+}}-T^{*} U$ is invertible, or equivalently, if and only if the $n_{+} \times n_{+}$matrix $I_{n_{+}}-T U^{*}$ is invertible, or equivalently, if and only if the $n_{-} \times n_{-}$matrix $I_{n_{-}}-U^{*} T$ is invertible, or equivalently, if and only if the $n_{-} \times n_{-}$matrix $I_{n_{-}}-U T^{*}$ is invertible, Suppose that this is the case, and let

$$
Z=\left(I_{n_{+}}-T U^{*}\right)^{-1}
$$

and

$$
Z^{\prime}=\left(I_{n_{+}}-T^{*} U\right)^{-1}
$$

Then the Hermitian matrix,

$$
\begin{aligned}
K=\psi-\psi C_{-} A_{-}^{-1} Z^{*} B_{-}+\psi C_{-} A_{-}^{-1} G_{-} U Z^{\prime} A_{-}^{*-1} C_{-}^{*} \psi \\
+B_{-}^{*} Z^{\prime} T^{*} G_{-}^{-1} B_{-}-B_{-}^{*} Z^{\prime} A_{-}^{*-1} C_{-}^{*} \psi
\end{aligned}
$$

is invertible and has $p$ positive and $q$ negative eigenvalues. Thus $K$ has a factorisation

$$
K=E^{*}\left(\begin{array}{cc}
I_{p} & 0 \\
0 & -I_{q}
\end{array}\right) E
$$

for an invertible matrix $E$. Then

$$
W(\lambda)=X_{+}^{*}(\lambda)\left(\begin{array}{cc}
I_{p} & 0 \\
0 & -I_{q}
\end{array}\right) X_{+}(\lambda)
$$

is a signed spectral factorisation of $W(\lambda)$ where

$$
\begin{array}{r}
X_{+}(\lambda)=E\left\{I_{m}+\left[-\psi^{-1} B_{-}^{*}\left(A_{-}^{\times}\right)^{*-1}+C_{-} U G_{-}^{*-1}\right] \cdot Z\left(\lambda G_{-}^{*-1}-A_{-}^{*-1}\right)^{-1}\right. \\
\left.\cdot\left[A_{-}^{*-1} C_{-}^{*} \psi-G_{-}^{*-1} T B_{-}\right]\right\}
\end{array}
$$

with inverse

$$
\begin{array}{r}
X_{+}(\lambda)^{-1}=\left\{I_{m}-\left[-\psi^{-1} B_{-}^{*}\left(A_{-}^{\times}\right)^{*-1}+C_{-} U G_{-}^{*-1}\right]\left[\lambda G_{-}^{*-1}-\left(A_{-}^{\times}\right)^{*-1}\right]^{-1}\right. \\
\left.. Z^{\prime}\left[A_{-}^{*-1} C_{-}^{*} \psi-G_{-}^{*-1} T B_{-}\right]\right\} E^{-1}
\end{array}
$$

Proof: We have that $W(\lambda)=Y_{+}(\lambda) Y_{-}(\lambda)$, where $Y_{+}(\infty)=Y_{-}(\infty)=I_{m}$. We consider $W(\infty)^{-1} W(\lambda)$ and its left canonical Wiener-Hopf factorisation with respect to $\mathbb{T}$. Then

$$
W(\infty)^{-1} W(\lambda)=Y_{+}(\lambda) Y_{-}(\lambda)=Y_{+}(\lambda)\left[Y_{-}(\infty)^{-1} Y_{-}(\lambda)\right]
$$


where

$$
Y_{+}(\lambda)=W(\infty)^{-1} Y_{-}^{*}(\lambda)\left(\begin{array}{cc}
I_{p} & 0 \\
0 & -I_{q}
\end{array}\right) Y_{-}(\infty)
$$

By hypothesis,

$$
Y_{-}(\infty)^{-1} Y_{-}(\lambda)=I_{m}+C_{-}\left(\lambda G_{-}-A_{-}\right)^{-1} B_{-},
$$

where $G_{-}, A_{-}, B_{-}$and $C_{-}$are given. Then we have that

$$
\begin{aligned}
Y_{-}^{*}(\lambda) & =Y_{-}\left(\frac{1}{\bar{\lambda}}\right)^{*} \\
& =\left[I_{m}+B_{-}^{*}\left(\lambda^{-1} G_{-}^{*}-A_{-}^{*}\right)^{-1} C_{-}^{*}\right] Y_{-}(\infty)^{*} \\
& =\left[I_{m}-B_{-}^{*} A_{-}^{*-1} C_{-}^{*}-B_{-}^{*} A_{-}^{*-1}\left(\lambda G_{-}^{*-1}-A_{-}^{*-1}\right)^{-1} A_{-}^{*-1} C_{-}^{*}\right] Y_{-}(\infty)^{*},
\end{aligned}
$$

and hence it is clear that

$$
W(\infty)=Y_{-}^{*}(\infty)\left(\begin{array}{cc}
I_{p} & 0 \\
0 & -I_{q}
\end{array}\right) Y_{-}(\infty)=\left(I_{m}-B_{-}^{*} A_{-}^{*-1} C_{-}^{*}\right) \psi
$$

Therefore, we compute that

$$
\begin{aligned}
Y_{+}(\lambda) & =W(\infty)^{-1} Y_{-}^{*}(\lambda)\left(\begin{array}{cc}
I_{p} & 0 \\
0 & -I_{q}
\end{array}\right) Y_{-}(\infty) \\
& =I_{m}-\psi^{-1}\left[I_{m}-B_{-}^{*} A_{-}^{*-1} C_{-}^{*}\right]^{-1}\left[B_{-}^{*} A_{-}^{*-1}\left(\lambda G_{-}^{*-1}-A_{-}^{*-1}\right)^{-1} A_{-}^{*-1} C_{-}^{*}\right] \psi
\end{aligned}
$$

Clearly, $W(\infty)^{-1} W(\lambda)$ has a right canonical Wiener-Hopf factorisation if and only if $W(\lambda)$ has one. By the remarks preceding this theorem, this is equivalent to the existence of a signed spectral factorisation for $W$.

To obtain conditions for a right canonical factorisation for $W(\infty)^{-1} W(\lambda)$ we apply Theorem 2.1 with $G_{-}, A_{-}, B_{-}$and $C_{-}$as given here, but with $G_{+}, A_{+}, B_{+}$and $C_{+}$ given by:

$$
\begin{array}{cl}
G_{+}=G_{-}^{*-1}, & A_{+}=A_{-}^{*-1} \\
B_{+}=A_{-}^{*-1} C_{-}^{*} \psi, & C_{+}=-\psi^{-1} B_{-}^{*}\left(A_{-}^{\times}\right)^{*-1} .
\end{array}
$$

Next, we compute the associate operator,

$$
\begin{aligned}
A_{+}^{\times} & =A_{+}-B_{+} C_{+} \\
& =A_{-}^{*-1}+A_{-}^{*-1} C_{-}^{*} B_{-}^{*}\left(A_{-}^{\times}\right)^{*-1} \\
& =\left(A_{-}^{\times}\right)^{*-1} .
\end{aligned}
$$


By replacing $G_{+}, A_{+}^{\times}$and $C_{+}$in the Lyapunov equation,

$$
A_{-}^{\times} U G_{+}-G_{-} U A_{+}^{\times}=B_{-} C_{+},
$$

with the expressions determined above, it is clear that (12) holds. Note that the solution $U$ of (12) is unique. Similarly, by replacing $G_{+}, A_{+}$and $B_{+}$in equation (7), we find that (13) holds and has the unique solution $T$. Also, from (12), we have

$$
A_{-}^{\times} U G_{-}^{*-1}\left(A_{-}^{\times}\right)^{*}-G_{-} U=-B_{-} \psi^{-1} B_{-}^{*}=A_{-}^{\times} G_{-}^{-1} U^{*}\left(A_{-}^{\times}\right)^{*}-U^{*} G_{-}^{*} ;
$$

Then, since (12) is uniquely solvable, it follows that

$$
U=G_{-}^{-1} U^{*} G_{-}^{*}
$$

Similarly, from (13), we have

$$
T=G_{-}^{*} T^{*} G_{-}^{-1}
$$

Furthermore, where $Z=\left(I_{n_{+}}-T U^{*}\right)^{-1}$ and $Z^{\prime}=\left(I_{n_{+}}-T^{*} U\right)^{-1}$, we conclude that

$$
T^{*} G_{-}^{-1} Z^{*}=Z^{\prime} T^{*} G_{-}^{-1}, \quad Z^{*} G_{-} U=G_{-} U Z^{\prime} .
$$

Thus the invertibility of $I_{n_{+}}-T^{*} U$, or equivalently, the invertibility of $I_{n_{+}}-T U^{*}$, or equivalently, the invertibility of $I_{n_{-}}-U^{*} T$, or equivalently, the invertibility of $I_{n_{-}}-U T^{*}$ is a necessary and sufficient condition for the existence of a signed spectral factorisation of $W(\lambda)$.

Then, by applying formulas (8)-(11) of Theorem 2.1 we have that

$$
W(\infty)^{-1} W(\lambda)=W_{-}(\lambda) W_{+}(\lambda)
$$

is a right canonical Wiener-Hopf factorisation, with

$$
\begin{array}{r}
W_{+}(\lambda)=I_{m}+\left[-\psi^{-1} B_{-}^{*}\left(A_{-}^{\times}\right)^{*-1}+C_{-} U G_{-}^{*-1}\right] \cdot Z\left(\lambda G_{-}^{*-1}-A_{-}^{*-1}\right)^{-1} \\
\cdot\left[A_{-}^{*-1} C_{-}^{*} \psi-G_{-}^{*-1} T B_{-}\right]
\end{array}
$$

and its inverse

$$
\begin{aligned}
W_{+}(\lambda)^{-1}=I_{m}-\left[-\psi^{-1} B_{-}^{*}\left(A_{-}^{\times}\right)^{*-1}+C_{-} U G_{-}^{*-1}\right] & \left(\lambda G_{-}^{*-1}-\left(A_{-}^{\times}\right)^{*-1}\right)^{-1} \\
. & Z^{\prime}\left[A_{-}^{*-1} C_{-}^{*} \psi-G_{-}^{*-1} T B_{-}\right] .
\end{aligned}
$$

In particular,

$$
W(\lambda)=W(\infty) W_{-}(\lambda) W_{+}(\lambda)
$$


is a right canonical Wiener-Hopf factorisation of $W$. Thus, the same is true for

$$
W(\lambda)=W^{*}(\lambda)=W_{+}^{*}(\lambda) W_{-}^{*}(\lambda) W^{*}(\infty)
$$

By the uniqueness of the right canonical factorisation, we know that there is a constant, invertible matrix $K$ such that

$$
W(\infty) W_{-}(\lambda)=W_{+}^{*}(\lambda) K
$$

Therefore,

$$
W(\lambda)=W_{+}^{*}(\lambda) K W_{+}(\lambda)
$$

By calculating both sides of (19) at a point $\lambda$ on the unit circle and using the original signed antispectral factorisation for $W$, we get that $K$ is invertible with $p$ positive and $q$ negative eigenvalues. Thus $K$ can be factored as

$$
K=E^{*}\left(\begin{array}{cc}
I_{p} & 0 \\
0 & -I_{q}
\end{array}\right) E
$$

for some invertible matrix $E$. Then (19) yields

$$
\begin{aligned}
W(\lambda) & =W_{+}^{*}(\lambda) E^{*}\left(\begin{array}{cc}
I_{p} & 0 \\
0 & -I_{q}
\end{array}\right) E W_{+}(\lambda) \\
& =X_{+}^{*}(\lambda)\left(\begin{array}{cc}
I_{p} & 0 \\
0 & -I_{q}
\end{array}\right) X_{+}(\lambda)
\end{aligned}
$$

which is a signed spectral factorisation of $W(\lambda)$, where $X_{+}(\lambda)=E W_{+}(\lambda)$. Employing formulas (17) and (18), we obtain the required formulas (15) and (16) for $X_{+}(\lambda)$ and $X_{+}(\lambda)^{-1}$, provided we verify that the constant $K$ in (19) is given by the formula (14).

To evaluate $K$, we set $\lambda=\infty$ in (19) to obtain

$$
K=W_{+}^{*}(\infty)^{-1} W(\infty)=W_{+}(0)^{*-1} W(\infty)
$$

From (18), we see that

$$
\begin{aligned}
W_{+}(0)^{*-1} & =\left[W_{+}(0)^{-1}\right]^{*} \\
& =I_{m}+\left[\psi C_{-} A_{-}^{-1}-B_{-}^{*} T^{*} G_{-}^{-1}\right] Z^{*}\left(A_{-}^{\times}\right) \cdot\left[-\left(A_{-}^{\times}\right)^{-1} B_{-} \psi^{-1}+G_{-}^{-1} U^{*} C_{-}^{*}\right] .
\end{aligned}
$$

We have already observed that

$$
W(\infty)=\left[I_{m}-B_{-}^{*} A_{-}^{*-1} C_{-}^{*}\right] \psi
$$


To compute the product $K=W_{+}(0)^{*-1} W(\infty)$, we first simplify the expression

$$
\left[-\left(A_{-}^{\times}\right)^{-1} B_{-} \psi^{-1}+G_{-}^{-1} U^{*} C_{-}^{*}\right] W(\infty)
$$

as follows

$$
\begin{aligned}
{[-} & \left.\left(A_{-}^{\times}\right)^{-1} B_{-} \psi^{-1}+G_{-}^{-1} U^{*} C_{-}^{*}\right]\left[\psi-B_{-}^{*} A_{-}^{*-1} C_{-}^{*} \psi\right] \\
= & -\left(A_{-}^{\times}\right)^{-1} B_{-}-\left(A_{-}^{\times}\right)^{-1}\left[A_{-}^{\times} U G_{-}^{*-1}\left(A_{-}^{\times}\right)^{*}-G_{-} U\right] A_{-}^{*-1} C_{-}^{*} \psi \\
& \quad+G_{-}^{-1} U^{*} C_{-}^{*}\left[\psi-B_{-}^{*} A_{-}^{*-1} C_{-}^{*} \psi\right] \\
& =\left(A_{-}^{\times}\right)^{-1}\left[-B_{-}+G_{-} U A_{-}^{*-1} C_{-}^{*} \psi\right]+\left[G_{-}^{-1} U^{*}-U G_{-}^{*-1}\right] C_{-}^{*}\left[\psi-B_{-}^{*} A_{-}^{*-1} C_{-}^{*} \psi\right] \\
= & \left(A_{-}^{\times}\right)^{-1}\left[-B_{-}+G_{-} U A_{-}^{*-1} C_{-}^{*} \psi\right]
\end{aligned}
$$

Here we have used the fact that $U=G_{-}^{-1} U^{*} G_{-}^{*}$. Thus, it follows that

$$
\begin{aligned}
K= & {\left[W_{+}(0)^{-1}\right]^{*} W(\infty) } \\
= & \psi-\psi C_{-} A_{-}^{-1} Z^{\prime *} B_{-}+\psi C_{-} A_{-}^{-1} G_{-} U Z^{\prime} A_{-}^{*-1} C_{-}^{*} \psi \\
& \quad+B_{-}^{*} Z^{\prime} T^{*} G_{-}^{-1} B_{-}-B_{-}^{*} Z^{\prime} A_{-}^{*-1} C_{-}^{*} \psi
\end{aligned}
$$

which coincides with (14). This completes the proof of the theorem.

The model reduction problem for discrete time systems (see [2]), suggests the application of Theorem 3.1 to a special form of the rational matrix function $Y_{-}(\lambda)$.

Corollary 3.2. Suppose $L(\lambda)=C(\lambda G-A)^{-1} B$ is a $p \times q$ rational matrix function of Mcmillan degree $n$ such that all the poles of $L$ are in the open unit disk $\mathbb{D}_{+}$. Thus we may assume that $\sigma(G, A) \subset \mathbb{D}_{+}$. For $\mu$ a positive real number, define the matrix function $W(\lambda)$ by

$$
W(\lambda)=\left(\begin{array}{cc}
I_{p} & 0 \\
L^{*}(\lambda) & \mu I_{q}
\end{array}\right)\left(\begin{array}{cc}
I_{p} & 0 \\
0 & -I_{q}
\end{array}\right)\left(\begin{array}{cc}
I_{p} & L(\lambda) \\
0 & \mu I_{q}
\end{array}\right)
$$

and let $U$ and $T$ be the unique solutions of the Stein equations

$$
A\left(\mu^{2} U\right) G^{*-1} A^{*}-G\left(\mu^{2} U\right)=B B^{*}
$$

and

$$
A^{*} G^{*-1} T A-T G=C^{*} C .
$$

Then $W$ has a signed spectral factorisation if and only if the matrix $I_{n}-T^{*} U$ is invertible, or equivalently, if and only if the matrix $I_{n}-T U^{*}$ is invertible, or equivalently, if and only if the matrix $I_{n}-U^{*} T$ is invertible, or equivalently, if and only if the matrix 
$I_{n}-U T^{*}$ is invertible. When this is the case, the factor $X_{+}(\lambda)$ for a signed spectral factorisation

$$
W(\lambda)=X_{+}^{*}(\lambda)\left(\begin{array}{cc}
I_{p} & 0 \\
0 & -I_{q}
\end{array}\right) X_{+}(\lambda)
$$

is computed as follows. Set

$$
Z=\left(I_{n}-T U^{*}\right)^{-1}
$$

and

$$
Z^{\prime}=\left(I_{n}-T^{*} U\right)^{-1}
$$

and let $K$ be the $(p+q) \times(p+q)$ matrix

$$
K=\left(\begin{array}{cc}
I+C A^{-1} G U Z^{\prime} A^{*-1} C^{*} & -C A^{-1} Z^{\prime *} B \\
-B^{*} Z^{\prime} A^{*-1} C^{*} & -\mu^{2} I_{q}+B^{*} Z^{\prime} T^{*} G^{-1} B
\end{array}\right) .
$$

Then $K$ is Hermitian with $p$ positive and $q$ negative eigenvalues, and so has the factorisation

$$
K=E^{*}\left(\begin{array}{cc}
I_{p} & 0 \\
0 & -I_{q}
\end{array}\right) E
$$

for an invertible $(p+q) \times(p+q)$ matrix $E$. Then the spectral factor $X_{+}(\lambda)$ for $W(\lambda)$ in this case is given by

$$
X_{+}(\lambda)=E\left\{\left(\begin{array}{cc}
I_{p} & 0 \\
0 & I_{q}
\end{array}\right)+\left(\begin{array}{c}
C U G^{*-1} \\
\mu^{-2} B^{*} A^{*-1}
\end{array}\right) Z\left(\lambda G_{-}^{*-1}-A_{-}^{*-1}\right)^{-1}\left[\begin{array}{ll}
A^{*-1} C^{*} & \left.-G^{*-1} T B\right]
\end{array}\right\}\right.
$$

with inverse given by

$$
\begin{aligned}
X_{+}(\lambda)^{-1}=\left\{\left(\begin{array}{cc}
I_{p} & 0 \\
0 & I_{q}
\end{array}\right)-\left(\begin{array}{c}
C U G^{*-1} \\
\mu^{-2} B^{*} A^{*-1}
\end{array}\right)\right. & \left.\left(\lambda G_{-}^{*-1}-A_{-}^{*-1}\right)^{-1} Z^{\prime}\left[A^{*-1} C^{*}-G^{*-1} T B\right]\right\} E^{-1} .
\end{aligned}
$$

Proof: The result follows from Theorem 3.1 above, if one takes

$$
\begin{aligned}
Y_{-}(\lambda) & =\left(\begin{array}{cc}
I_{p} & 0 \\
0 & \mu I_{q}
\end{array}\right)\left(\begin{array}{cc}
I_{p} & L(\lambda) \\
0 & I_{q}
\end{array}\right) \\
& =\left(\begin{array}{cc}
I_{p} & 0 \\
0 & \mu I_{q}
\end{array}\right)\left[\left(\begin{array}{cc}
I_{p} & 0 \\
0 & I_{q}
\end{array}\right)+\left(\begin{array}{c}
C \\
0
\end{array}\right)(\lambda G-A)^{-1}\left(\begin{array}{ll}
0 & B
\end{array}\right)\right] .
\end{aligned}
$$

Note that then both $Y_{-}(\lambda)$ and

$$
Y_{-}(\lambda)^{-1}=\left(\begin{array}{cc}
I_{p} & -L(\lambda) \\
0 & I_{q}
\end{array}\right)\left(\begin{array}{cc}
I_{p} & 0 \\
0 & \mu^{-1} I_{q}
\end{array}\right)
$$

are analytic on the complement of the unit disk $\mathbb{D}_{+}$since all the poles of $L(\lambda)$ are assumed to be in $\mathbb{D}_{+}$. 


\section{REFERENCES}

[1] J.A. Ball and A.C.M. Ran, 'Hankel norm approximation of a rational matrix function in terms of its realization', in Modelling, identification and robust control, (C.I. Byrnes and A. Lindquist, Editors) (North Holland, Amsterdam, 1986).

[2] J.A. Ball and A.C.M. Ran, 'Optimal Hankel norm model reductions and Wiener-Hopf factorization I: The canonical case', SIAM J. Control Optim. 25 (1987), 362-382.

[3] J.A. Ball and A.C.M. Ran, 'Left versus right canonical Wiener-Hopf factorization', in Constructive methods of Wiener-Hopf factorization, (I. Gohberg and M.A. Kaashoek, Editors), Operator theory: advances and applications 21 (Birkhäuser Verlag, Basel, 1986), pp. $9-37$.

[4] H. Bart, I. Gohberg and M.A. Kaashoek, Minimal factorization of matrix and operator functions, Operator theory: advances and applications 1 (Birkhäuser Verlag, Basel, 1979).

[5] H. Bart, I. Gohberg and M.A. Kaashoek, 'The state space method in problems of analysis', in Proc. 1st Int. Conf. on Ind. and Appl. Math. (Centrum Wisk. Inform., Amsterdam, 1987), pp. 1-16.

[6] K. Clancey and I. Gohberg, Factorization of matrix functions and singular integral operators, Operator theory: advances and applications 3 (Birkhäuser Verlag, Basel, 1981).

[7] B.A. Francis, $A$ course in $H_{\infty}$ control (Springer Verlag, New York, 1987).

[8] I. Gohberg, S. Goldberg and M.A. Kaashoek, Classes of linear operators, Vol II, Operator theory: advances and applications 63 (Birkhäuser Verlag, Basel, 1993).

[9] I. Gohberg and M.A. Kaashoek, 'Block Toeplitz operators with a rational symbol', in Contributions to operator theory, systems and networks, (I. Gohberg, J.W. Helton and L. Rodman, Editors), Operator theory: advances and applications 35 (Birkhäuser Verlag, Basel, 1988), pp. 385-440.

[10] I. Gohberg and M.G. Krein, 'Systems of integral equations on a half-line with kernels depending on the difference arguments', (in Russian), Uspekhi Mat. Nauk 13 (1958), 3-72, (Amer. Math. Soc. Transl. 14, (1960), 217-287).

[11] M. Green, K. Glover, D.J.N. Limebeer and J. Doyle, 'A J-spectral factorization approach to $H_{\infty}$ control', SIAM J. Control Optim. 28 (1990), 1350-1371.

[12] G.J. Groenewald and M.A. Petersen, 'Left and right symmetric spectral factorization of rational matrix functions and realization', (submitted).

[13] G.J. Groenewald, M.A. Petersen and Y. Zucker, 'Left versus right canonical Wiener-Hopf factorization and realization', Integral Equations Operator Theory (to appear).

[14] J.W. Helton, 'A spectral factorization approach to the distributed stable regulator problem: the algebraic Riccati equation', SIAM J. Control Optim. 14 (1976), 639-661.

[15] J. Willems, 'Least squares stationary optimal control and the algebraic Riccati equation', IEEE Trans. Automat. Control 16 (1971), 621-634.

Department of Mathematics and Applied Mathematics

University of the Western Cape

Private Bag X17

Bellville 7535

South Africa 